\title{
Covid-19 Digitally enhanced recovery from severe COVID-19: a new frontier?
}

\author{
Authors: Alexander Hunter, ${ }^{A}$ Todd Leckie, ${ }^{\mathrm{B}}$ Daniel Fitzpatrick, ${ }^{\mathrm{C}}$ Anacarolina Goncalves, ${ }^{\mathrm{D}}$ Alan Richardson ${ }^{\mathrm{E}}$ and \\ Luke Hodgson ${ }^{F}$
}

During the first wave of intensive care unit admissions with COVID-19, in response to the constraints of social distancing we introduced a new digitally enabled critical care rehabilitation pathway. Using smartwatch technology, this pathway rapidly enabled our multidisciplinary team to observe the recovery of a COVID-19 cohort across eight NHS acute hospitals across the south of England. This represents one of the geographically largest smartwatch studies of its kind.

KEYWORDS: digital assessment, digital rehabilitation, COVID-19, smartwatch technology

DOI: $10.7861 /$ fhj.2020-0271

\section{Introduction}

The global COVID-19 pandemic has resulted in a large cohort of survivors of severe acute lung injury. In the immediate period following the first surge of admissions to UK hospitals, social distancing and local policies made usual face-to-face follow-up and social network support at best attenuated. This created a unique opportunity for digital innovation.

The potential for using smartphones/wearable technology in health is well recognised, ${ }^{1}$ with feasibility assessed in a growing number of studies, ${ }^{2,3}$ particularly in chronic diseases. ${ }^{4}$ However, few studies have developed a model for integrating this technology at a local level and to date we could find no reporting of their use in a COVID-19 cohort. In this study we report the integration of Fitbit ${ }^{\mathrm{TM}}$ smartwatches to observe recovery from severe COVID-19 and, at one site, to improve the rehabilitation pathway from discharge to one year via a remote multi-disciplinary team (MDT).

\section{Smartwatches used to observe recovery}

Adults ( $\geq 18$ year of age) from eight sites located in the south of England who had required invasive positive pressure ventilation

Authors: Aintensive care registrar, Worthing Hospital, Worthing, UK; ${ }^{B}$ anaesthetic registrar, Brighton University Hospitals, Brighton, UK; Chonorary fellow, University of Brighton, Brighton, UK; ${ }^{D}$ senior physiotherapist, Worthing Hospital, Worthing, UK; Esenior lecturer, University of Brighton, Brighton, UK; Fintensive care and respiratory consultant, Worthing Hospital, Worthing, UK and visiting senior lecturer, University of Surrey, Guildford, UK or non-invasive ventilation as a result of confirmed COVID-19 and had at least a moderate lung injury $\left(\mathrm{PaO}_{2} / \mathrm{FiO}_{2}\right.$ ratio $\left.\leq 26.6 \mathrm{kPa}^{5}\right)$ were recruited at discharge from critical care, or shortly following discharge. At all sites, a comprehensive observational dataset and biopsychosocial measures were collected at hospital discharge; ${ }^{6}$ this was repeated at face-to-face sessions 3 months post-discharge and will be performed again at 1-year follow-up. Patients were provided with Fitbit Charge 4 smartwatches for the study period. These were set up by a study team member if required and written information was given to the patient regarding the use of the device. Data were extracted by the study team via the Fitbit app. Data collected from the smartwatches included step counts, resting heart rate, sedentary minutes and specific activities (eg running, cycling).

\section{Smartwatch enabled rehabilitation and the remote MDT}

At one site, an MDT of clinicians, physiotherapists and physiologists have met monthly and used retrieved smartwatch data to set goals and track recovery (Fig 1), using closed loop feedback to promote physical recovery. ${ }^{7}$ Initial exercise programmes were set based on the discharge measures collected, which included individual illness severity, age and performance on physical discharge assessments (incremental shuttle walk [ISWT] ${ }^{8}$, Chelsea critical care physical assessment $[\mathrm{CPAx}]^{9}$ and 1 -minute sit-to-stand). Exercise plans were reviewed and tailored by the remote MDT, supported by video or phone discussions with the patients. Patients received at least monthly personal follow-up in the form of a phone call from a member of the MDT. Fitbit data were downloaded on a monthly basis ahead of the MDT meeting and technical issues with the Fitbit watches were addressed by the research fellow as soon as possible.

At the MDT site a locally designed dashboard that pulled data from the Fitbit app was set up to facilitate these team meetings. This allowed each patient from the cohort to be discussed in turn with their Fitbit data readily available (Fig 2).

\section{Outcomes}

60 patients were recruited from April to June 2020, aged 35-77, with 54 receiving smartwatches. The smartwatches have provided a rich real-time dataset that has allowed monitoring of this population's recovery during the ongoing pandemic. Six patients did not receive watches either due to patient preference 


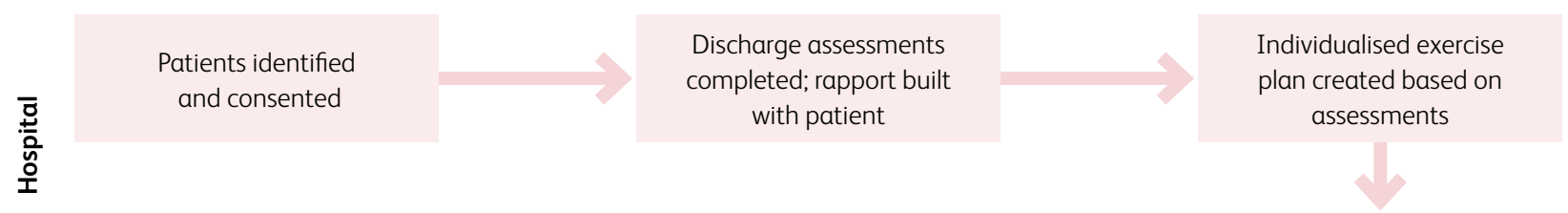

Smartwatch delivered as inpatient or to home if discharged; patient informed in features and usage of smartwatch

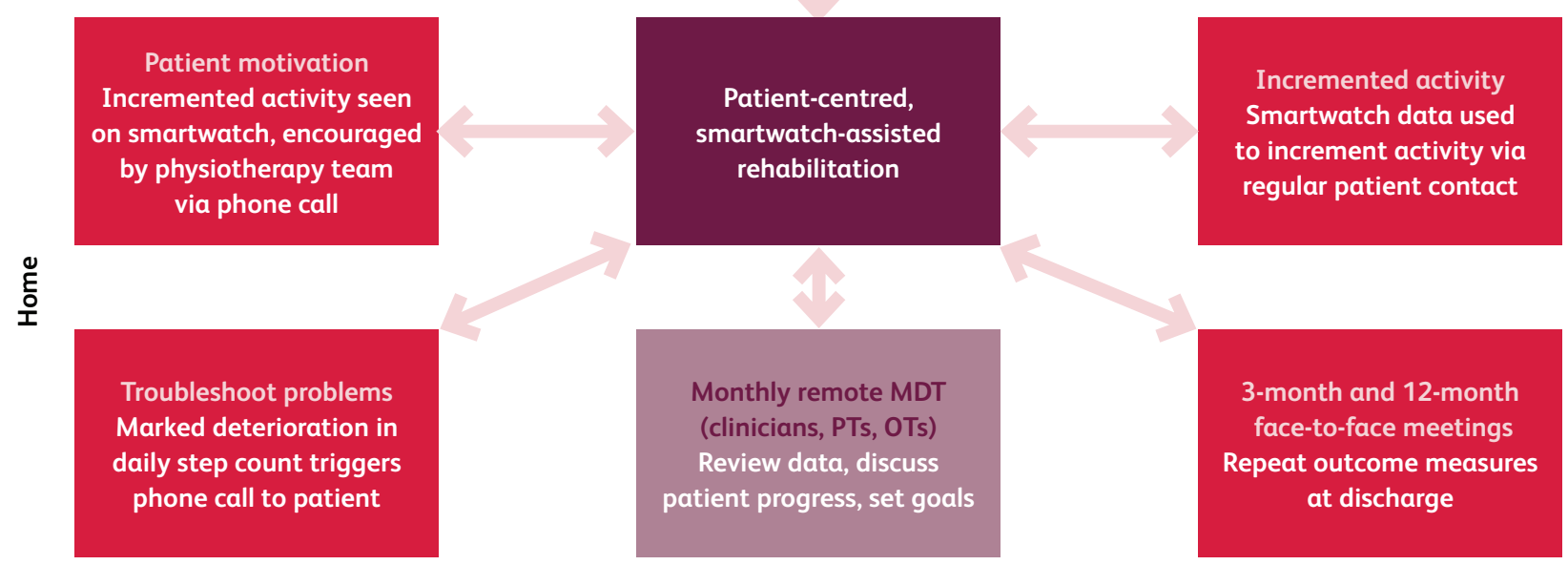

Fig 1. Implementation of socially enhanced rehabilitation pathway at one site. MDT = multidisciplinary team meeting; OT = occupational therapist; PT $=$ physiotherapist.

or technical issues such as the patient not having a compatible smartphone. As with any new pathway, there are a number of implementation issues and ongoing improvements discussed below.

The smartwatches were able to detect an improvement in step count, heart rate and sedentary minutes following hospital discharge. This was rewarding to witness in patients following severe lung injuries. The cohort reviewed thus far $(n=27)$ on average increased daily steps from 4,268 (standard deviation [SD] $3,926)$ in the first 2 weeks of data collected post hospital discharge

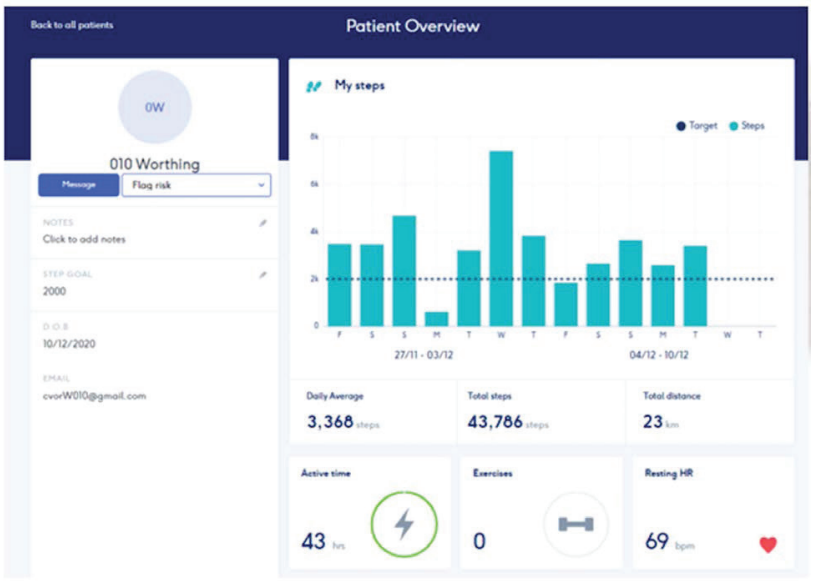

Fig 2. Example patient dashboard. to $6,189(\mathrm{SD} 4,420)$ at 3-month follow-up ( $<<0.05)$. Resting heart rate slowed from 78 (SD 8) post-discharge to 70 (SD 5) at followup $(p<0.05)$. Daily sedentary minutes per day declined from 903 (SD 311) at discharge to 842 (SD 306) at follow-up ( $p<0.05)$. Patient engagement and acceptability with the technology was generally good. Of patients surveyed at follow up (Fig 3), 78\% of patients reported wearing their device daily, and $75 \%$ reported that the device was easy to use. Subjective assessment of whether the watches helped with recovery or motivated the patients provided was more varied; however, $>50 \%$ of the cohort agreed with the statement that 'the device has helped and motivated my recovery'. $80 \%$ of patients reported aiming to increase their activity level over time.

With the MDT cohort ( $n=17$ ), aged 42-73, the technology created a unique relationship. The smartwatch-assisted programme created a shared rehabilitation journey with the clinical team, which we believe provided insight into patient recovery beyond face-to-face interaction alone. This contrasts with the often long, sometimes lonely course to recovery following discharge with a severe illness. ${ }^{10}$ Through the remote MDT and step count data review, the MDT could view patients struggling with their recovery promptly. This, for example, would usually be followed by a phone call from a member of the team to enquire about whether a particular problem needed addressing. The types of problems identified in this way included musculoskeletal complaints, dizziness/balance disturbance, cardiac symptoms and non-improving shortness of breath. These prompted six out of 17 patients to be referred to services outside the immediate scope of the MDT, including cardiac rehabilitation $(n=1)$, specialist physiotherapy $(n=2)$, further radiological investigations (CT scan, 

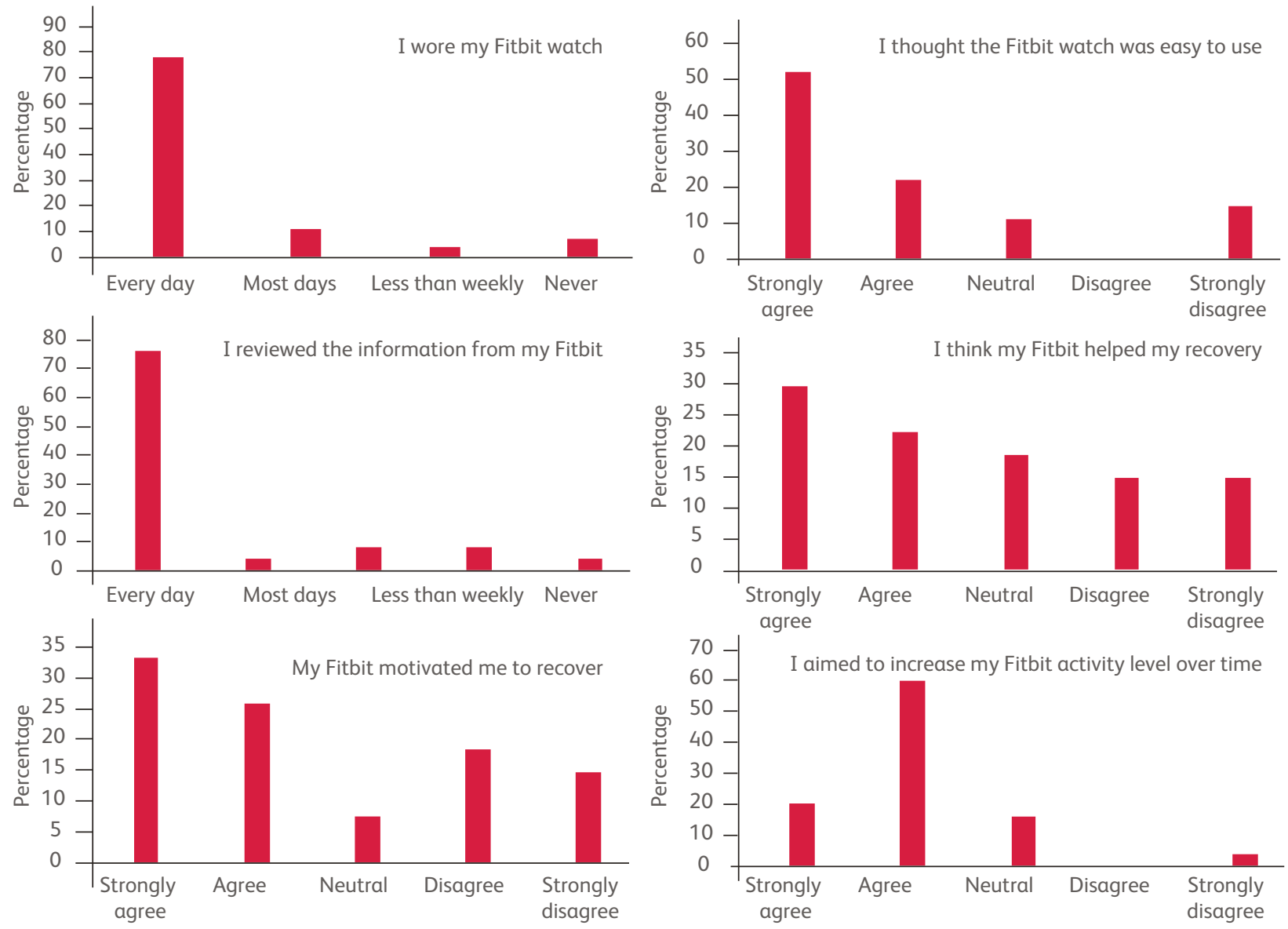

Fig 3. Smartwatch engagement collected at 3-month follow up. $n=27$.

$n=1)$ or other services $(n=2)$. Similarly, consistent increases in daily step count were safely encouraged by closed loop feedback from the physiotherapy team. Patients were moved up to a higher level on the exercise programme after discussion and consensus agreement at the MDT meeting.

\section{Problems}

A minority of the cohort had little engagement with the smartwatches. This was a small but significant number (three out of 17 patients from the MDT cohort). A relatively common issue has been not syncing the smartwatches. A number of the devices were set up by the study team and patients often needed to be reminded to open the app and sync the smartwatch. This could be done via phone call or during one of the face-to-face meetings. Interestingly most patients with little or no experience using such devices found the process straightforward to achieve and regularly uploaded data. One watch has broken; however, generally the watches were durable.

The nature of the project involved a sizeable study team. While this was essential for the study, rolling out a larger smartwatchassisted programme is a significant resource pull and could impact on feasibility. Setting up and maintaining the data was predominantly managed by the regular physiotherapy team and a research clinical fellow. The workload reduced significantly after the initial process of set-up. The watches were funded for this project, though per unit they are relatively cheap (£70). Patients required a smartphone to sync the watch, though this excluded only one recruited patient. A limited number of smartphones failed to communicate with the Fitbit app, excluding two patients. Clearly such teething issues would need to be factored in for centres wishing to adopt a similar approach or if a large randomised controlled trial of the technology-enabled intervention was being considered.

\section{Conclusion}

To our knowledge this is the first report of a technologyenabled pathway following severe COVID-19. Feasibility has been investigated at multiple centres with good acceptability reported by patients and clinical teams. The work has provided real-world insights and we intend to expand the pathway to all our critical care discharges. This is a relatively small, albeit multi-centre observational study and randomised control trials are needed to determine the efficacy of smartwatch-assisted technology in helping patients' recovery processes. Future work will involve integrating smartwatch technology beyond a critical care population to include hip fracture patients and perioperative patients undergoing major elective surgery. We would welcome correspondence from groups with similar aims. 


\section{Acknowledgements}

This work would not have been possible without the help of the physiotherapy team at WSHFT - M Standing, A Palmer, K Cooper, G Stoner and D Geere - and our research department team - Koulouglioti C and Cox V. Funding was received from the University of Brighton and the British Association of Sports and Exercise Medicine.

\section{References}

1 Patel S, Park H, Bonato P, Chan L, Rodgers M. A review of wearable sensors and systems with application in rehabilitation. J Neuroeng Rehabil 2012;9:21.

2 Reeder B, David A. Health at hand: A systematic review of smartwatch uses for health and wellness. J Biomed Inform 2016;63: 269-76.

3 Baram S. Trimodal prehabilitation programme using smartwatches and mobile applications in patients undergoing major abdominal surgery: a randomised controlled pilot study. https://clinicaltrials. gov/ct2/show/NCT04047524.

4 Wu R, Liaqat D, de Lara $\mathrm{E}$ et al. Feasibility of using a smartwatch to intensively monitor patients with chronic obstructive pulmonary disease: prospective cohort study. JMIR Mhealth Uhealth 2018;6:e10046.
5 Ferguson ND, Fan E, Camporota L et al. The Berlin definition of ARDS: an expanded rationale, justification, and supplementary material. Intensive Care Med 2012;38:1573-82.

6 Marshall JC, Murthy S, Diaz ] et al. A minimal common outcome measure set for COVID-19 clinical research. Lancet Infect Dis 2020:20:e192-7.

7 Kramer JN, Kowatsch T. Using feedback to promote physical activity: the role of the feedback sign. J Med Internet Res 2017; 19:e192.

8 Singh SJ, Morgan M, Scott S, Walters D, Hardman AE. Development of a shuttle walking test of disability in patients with chronic airways obstruction. Thorax 1992;47:1019-24.

9 Corner EJ, Wood H, Englebretsen C et al. The Chelsea critical care physical assessment tool (CPAx): validation of an innovative new tool to measure physical morbidity in the general adult critical care population; an observational proof-of-concept pilot study. Physiotherapy 2013;99:33-41.

10 Wade DT. Rehabilitation after COVID-19: an evidence-based approach. Clin Med 2020;20:359-65.

Address for correspondence: Dr Alexander Hunter, Worthing Hospital, Lyndhurst Road, Worthing, West Sussex BN11 2DH, UK. Email: alexander.hunter2@nhs.net 